\title{
A Porous Metal-Organic Framework as an Electrochemical Sensing Platform for Highly Selective Adsorption and Detection of Bisphenols
}

Chang Liu, ${ }^{\perp,}$, Ze-Chen Sun $^{\perp},{ }^{\dagger}$ Wen-Yuan Pei ${ }^{\dagger}$, Jin Yang,,$\stackrel{*}{\dagger}$ Hong-Liang Xu ${ }^{\ddagger}$, Jing-Ping Zhang §, and Jian-Fang Ma* ${ }^{*}$

${ }^{\dagger}$ Key Laboratory of Polyoxometalate and Reticular Material Chemistry of Ministry of Education, Faculty of Chemistry, Northeast Normal University, Changchun, Jilin 130024, China

* Institute of Functional Material Chemistry, National \& Local United Engineering Lab for Power Battery, Key Laboratory of Polyoxometalate Science of Ministry of Education, Faculty of Chemistry, Northeast Normal University, Changchun, Jilin 130024, China

$\S$ Faculty of Chemistry, Northeast Normal University, Changchun, Jilin 130024, China

${ }^{\perp}$ C. L. and Z.-C. S. contributed equally

${ }^{*}$ Corresponding author

E-mail: yangj808@nenu.edu.cn (J. Yang). 
E-mail: majf247@nenu.edu.cn (J.-F. Ma).

\section{S1. Chemicals and Instruments}

Phosphate buffer solutions (PBS) $(0.1 \mathrm{M})$ of different $\mathrm{pH}$ values were achieved by disposing the stock $\mathrm{H}_{3} \mathrm{PO}_{4}$ solution $(0.1 \mathrm{M})$, and then their $\mathrm{pH}$ values were adjusted with $\mathrm{NaOH}$. Other chemicals were purchased.

Powder X-ray diffraction (PXRD) pattern was recorded on a Rigaku SmartLab $\mathrm{X}$-ray diffractometer with graphite monochromatized $\mathrm{Cu} \mathrm{K} \alpha$ radiation $(\lambda=0.154 \mathrm{~nm})$. Philips XL-30 ESEM was used to measure scanning electron microscopy (SEM) images. Elemental analyzer (EA) data was determined on a Euro vector EA3000 elemental analyzer. Thermogravimetric analysis (TGA) curve was recorded on a Perkin-Elmer Model TG-7 analyzer. FT-IR spectra were determined on a Nicolet 6700 FT-IR spectrometer with a KBr plate. Surface analysis of the catalyst particles was conducted by X-ray photoelectron spectroscopy (XPS) on an ESCA LAB spectrometer (USA) with a monochromatic $\mathrm{Al} \mathrm{K} \alpha$ source (hv $1486.6 \mathrm{eV}$ ). $\mathrm{N}_{2}$ adsorption-desorption isotherms were recorded by an Autosorb-iQ instrument. UV-Vis spectra were obtained by UV-Vis-NIR Spectrophotometer (Cary7000). HPLC spectra were conducted with Agilent-1220. ESI-MS spectra were measured by BRUKER micrOTOF II. Electrochemical behaviors were recorded on a CHI660E (Chenhua, Shanghai) electrochemical workstation with a conventional three-electrode system. The $\mathrm{GCE}, \mathrm{Ag} / \mathrm{AgCl}$ (in saturated $\mathrm{KCl}$ solution) and platinum wire were used as the working electrodes, reference electrode and counter electrode, respectively.

\section{S2. Synthesis of $\mathbf{L}^{1}$}

As shown in Scheme S1, the synthesis of $\mathbf{L}^{1}$ contains four steps. Compounds (I) and (II) were synthesized according to the known method. ${ }^{\mathrm{S} 1, \mathrm{~S} 2}$ The $30 \% \mathrm{H}_{2} \mathrm{O}_{2}(9.0 \mathrm{ml}$, $78.40 \mathrm{mmol})$ was added to the white powder (II) $(1.85 \mathrm{~g}, 1.8 \mathrm{mmol})$ in $\mathrm{CHCl}_{3}(100$ $\mathrm{ml})$ and $\mathrm{CF}_{3} \mathrm{CO}_{2} \mathrm{H}(15.0 \mathrm{ml})$. The resulting mixture was stirred at $298 \mathrm{~K}$ for $60 \mathrm{~h}$, and neutralized with a saturated aqueous solution of $\mathrm{NaHCO}_{3}$. The organic layer was separated and washed with brine, and dried with anhydrous $\mathrm{Na}_{2} \mathrm{SO}_{4}$. The white solid of compound (III) was achieved by removing the solvent under the reduced pressure 
and by recrystallization from $\mathrm{CH}_{2} \mathrm{Cl}_{2} / \mathrm{CH}_{3} \mathrm{OH}$ (yield 95\%). Further, a mixture of $\mathrm{NaOH}(1.60 \mathrm{~g}, 40 \mathrm{mmol})$ in dry DMF $(150 \mathrm{~mL})$ and imidazole $(0.68 \mathrm{~g}, 10.0 \mathrm{mmol})$ was stirred at $90^{\circ} \mathrm{C}$. After no white particles were found, compound (III) (4.73 g, 4.1 mmol) was added to the mixture and stirred at $90{ }^{\circ} \mathrm{C}$ for $12 \mathrm{~h}$. The solvent was evaporated and the residue was diluted with distilled water $(500 \mathrm{~mL})$ and filtrated. After washing the crude residue with distilled water $(100 \mathrm{~mL}), \mathbf{L}^{\mathbf{1}}$ was obtained as a white powder.

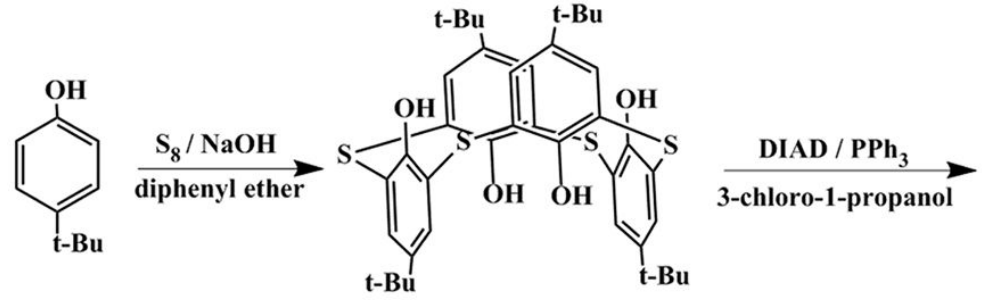

(I)

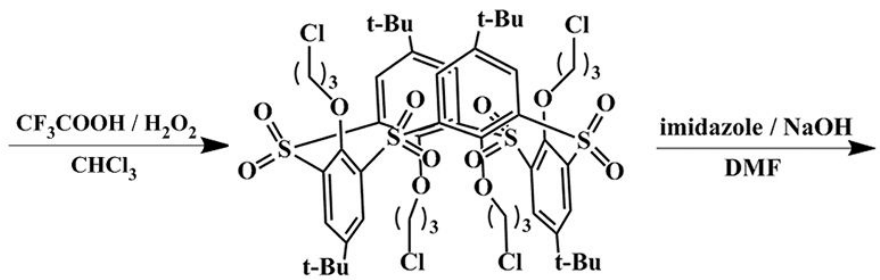

(III)

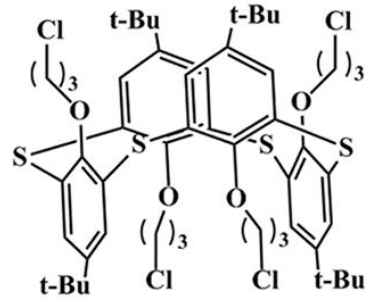

(II)

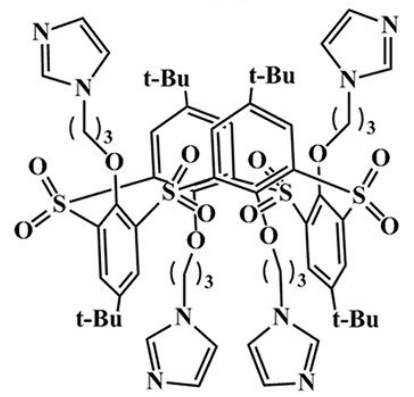

$\left(\mathrm{L}^{1}\right)$

Scheme S1. Synthetic route of $\mathbf{L}^{1}$ ligand.

\section{S3. Crystallographic Data Collection and Refinement}

Crystallographic data collection was conducted on a Bruker D8-Venture diffractometer with a Turbo X-ray Source $(\mathrm{Cu} \mathrm{K \alpha}$ radiation, $\lambda=0.71073 \AA)$ at $173 \mathrm{~K}$. The data frame was collected using the program APEX 3 and processed using the program SAINT routine in APEX 3. The structure was solved by direct method and refined by the full1matrix least-square on $F^{2}$ using the SHELXL-2018/3 program. ${ }^{\mathrm{S} 3, \mathrm{~S} 4}$ The solvent molecules were removed from the data with SQUEEZE routine of PLATON and refined using the data generated. The restrained SIMU, DFIX and ISOR instructions were used to refine the structure. The formula unit was obtained through a combination of elemental analyses thermogravimetric characterization and 
electron diffraction density.

\section{S4. Electrochemical Test}

Glass carbon electrode (GCE) was polished with 1.0, 0.3 and $0.05 \mu \mathrm{m}$ alumina slurry to a mirror and ultrasonically washed with 1:1 nitric acid, absolute alcohol and deionized water, respectively. Further, the GCE was rinsed with deionized water and dried in air. The modified Zn-L-IPA@GP/GCE was achieved by grinding Zn-L-IPA@GP (50 mg) and graphite powder (50 mg) for $30 \mathrm{~min}$. Afterwards, the as-synthesized sample $(3 \mathrm{mg})$ was dispersed into $1.0 \mathrm{~mL}$ Nafion $(0.5 \mathrm{wt} \%)$ under ultrasonication for $10 \mathrm{~min}$, until a homogenous dispersion was gained. The resulting suspension $(5.0 \mu \mathrm{L})$ was dropped on the GCE surface and dried under an infrared lamp. Zn-L-IPA/GCE and GP/GCE were obtained via the similar approach.

Cyclic voltammetry $(\mathrm{CV})$ curves were determined at a scanning rate of $50 \mathrm{mV} / \mathrm{s}$ from 0.0 to $1.0 \mathrm{~V}$ and amperometric i-t tests were used for accumulation steps. Differential pulse voltammetry (DPV) curves were obtained at the pulse amplitude of $50 \mathrm{mV}$, the scanning power of $10 \mathrm{mV} / \mathrm{s}$ with a step potential of $4 \mathrm{mV}$, and the pulse period of 0.5 s. EIS data was achieved through measuring the resistance of the interfacial charge-transfer of different electrode surfaces. In the frequency range of $0.1 \mathrm{~Hz}-10 \mathrm{kHz}$, the perturbation signal is $5 \mathrm{mV}$. Before the determination, the electrolytic solution was completely purged with nitrogen for 30 min to remove the dissolved oxygen interference in the solution.

\section{S5. Computational Methods}

Model Construction. The Zn-L-IPA supercell host structure was imported into AutoDock Tools-1.5.6 by adding hydrogen atoms, calculating charge and adjusting the atomic type. ${ }^{\mathrm{S} 5}$ Then bisphenol molecules were successively imported into AutoDock Tools-1.5.6. Atom type was assigned and atom charge was added. All flexible bonds were rotatable by default. ${ }^{\mathrm{s} 6}$

Molecular Docking. AutoDock Tools-1.5.6 was used for all docking. In the calculation process, $38 \times 38 \times 66$ lattice module with 0.375 grid was adopted, and the center was set $(21.779,21.011,15.686)$. Lamarckian genetic algorithm was applied 
for molecular docking calculation. The algorithm was as follows: maximum energy assessment of 25 million times, maximum number of 2000 times, crossover rate of 0.8 , mutation rate of $0.02,150$ population and independent docking operation of 50 times. The final docking structure was evaluated based on the binding energy. Pymol 2.3 software was employed for the visualization of docking results. ${ }^{\mathrm{S} 7}$

Deformation Energy Calculation. Calculation method was retrieving the conformation with the lowest binding energy from all the obtained conformations after docking between Zn-L-IPA and guests. The guest molecule structures were optimized under B3LYP/6-31G(d, p) basis set level to convergence. The energy and conformation of molecule structure will change during docking with Zn-L-IPA receptor. The deformation energy $\left(\Delta E_{\mathrm{d}}\right)$ of the molecule structure can be obtained from Equation S1

$$
\Delta E_{\mathrm{d}}=E_{\text {guest-sp }}-E_{\text {guest-opt }}
$$

The $E_{\text {guest-sp }}$ represents the electronic energy of the bisphenol guest in the host-guest model, and $E_{\text {guest-opt }}$ is the electronic energy of the relaxation bisphenol. ${ }^{\mathrm{S} 8}$ Both of these energies were conducted with Gaussian 09 software package based on B3LYP/6-31G(d, p) level. ${ }^{\text {S9,S10 }}$

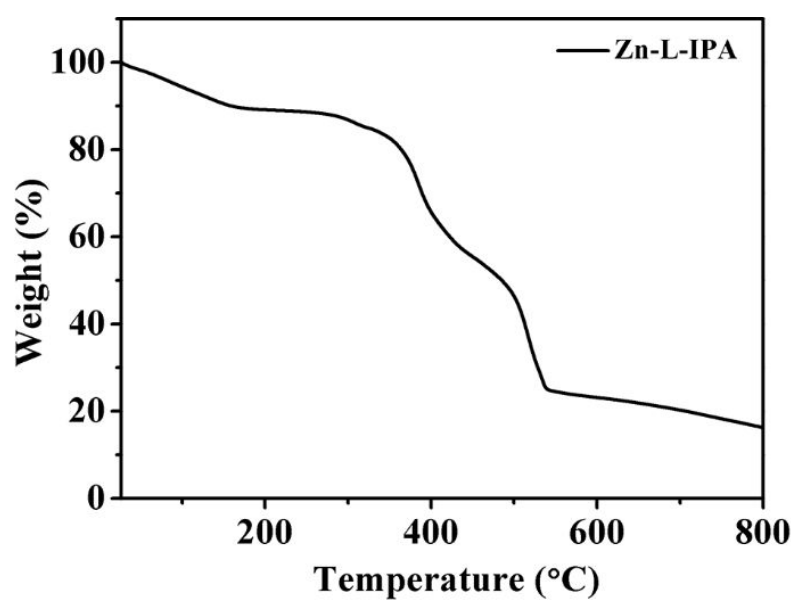

Figure S1. Thermogravimetric curve for Zn-L-IPA under nitrogen gas. The weight loss of $17.19 \%$ before $353{ }^{\circ} \mathrm{C}$ corresponded to the loss of one coordination water molecule, two free DMF molecules, two free methanol molecules and three free water 
molecules.

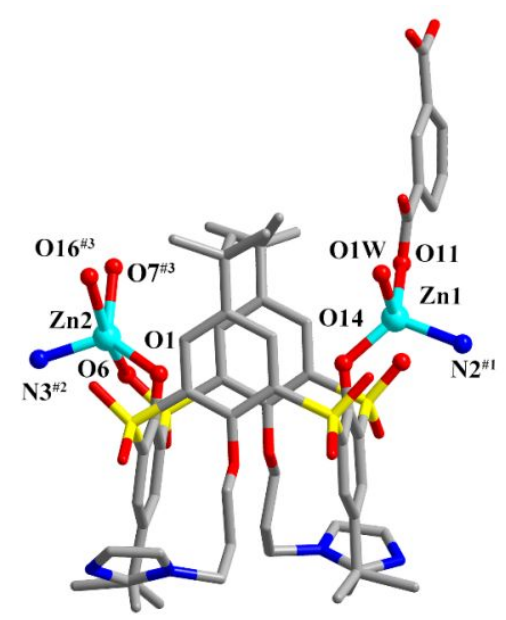

Figure S2. Coordination environments of $\mathrm{Zn}$ cations in Zn-L-IPA.

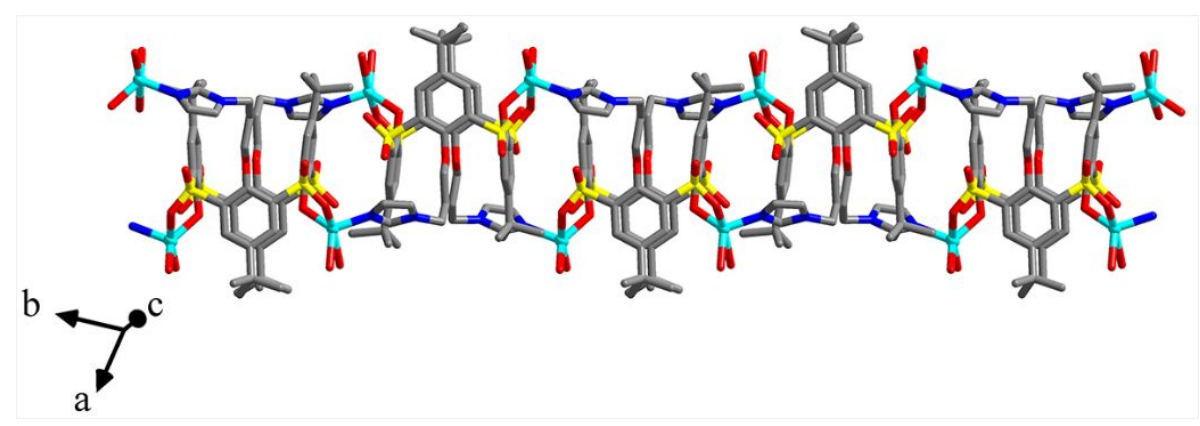

Figure S3. Chain structure in Zn-L-IPA. 


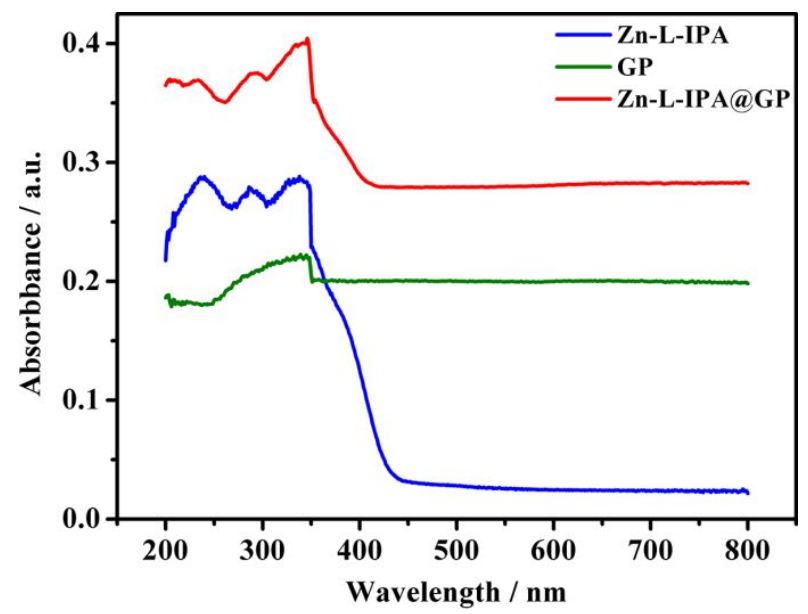

Figure S4. UV-vis spectra of Zn-L-IPA, GP and Zn-L-IPA@GP.

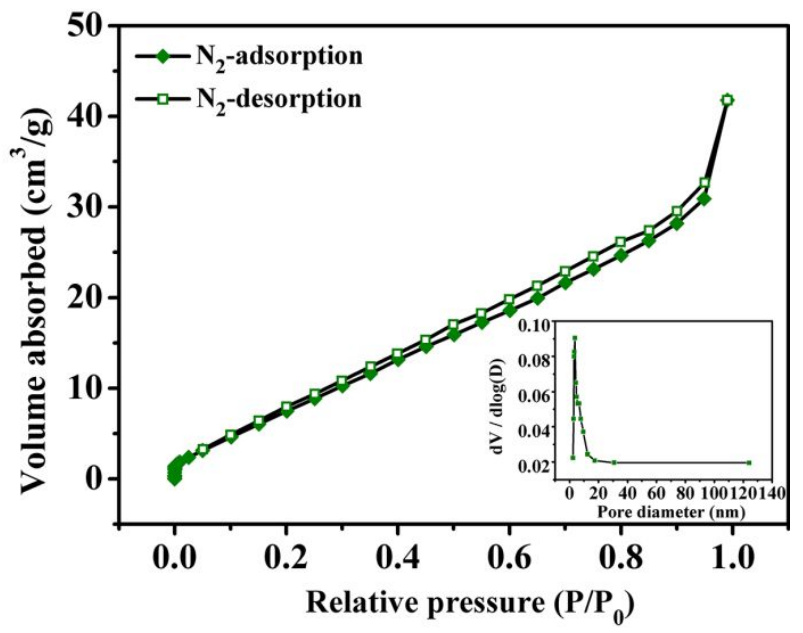

Figure S5. $\mathrm{N}_{2}$ adsorption-desorption isotherm of GP. Inset: corresponding BJH pore size distribution curve. 

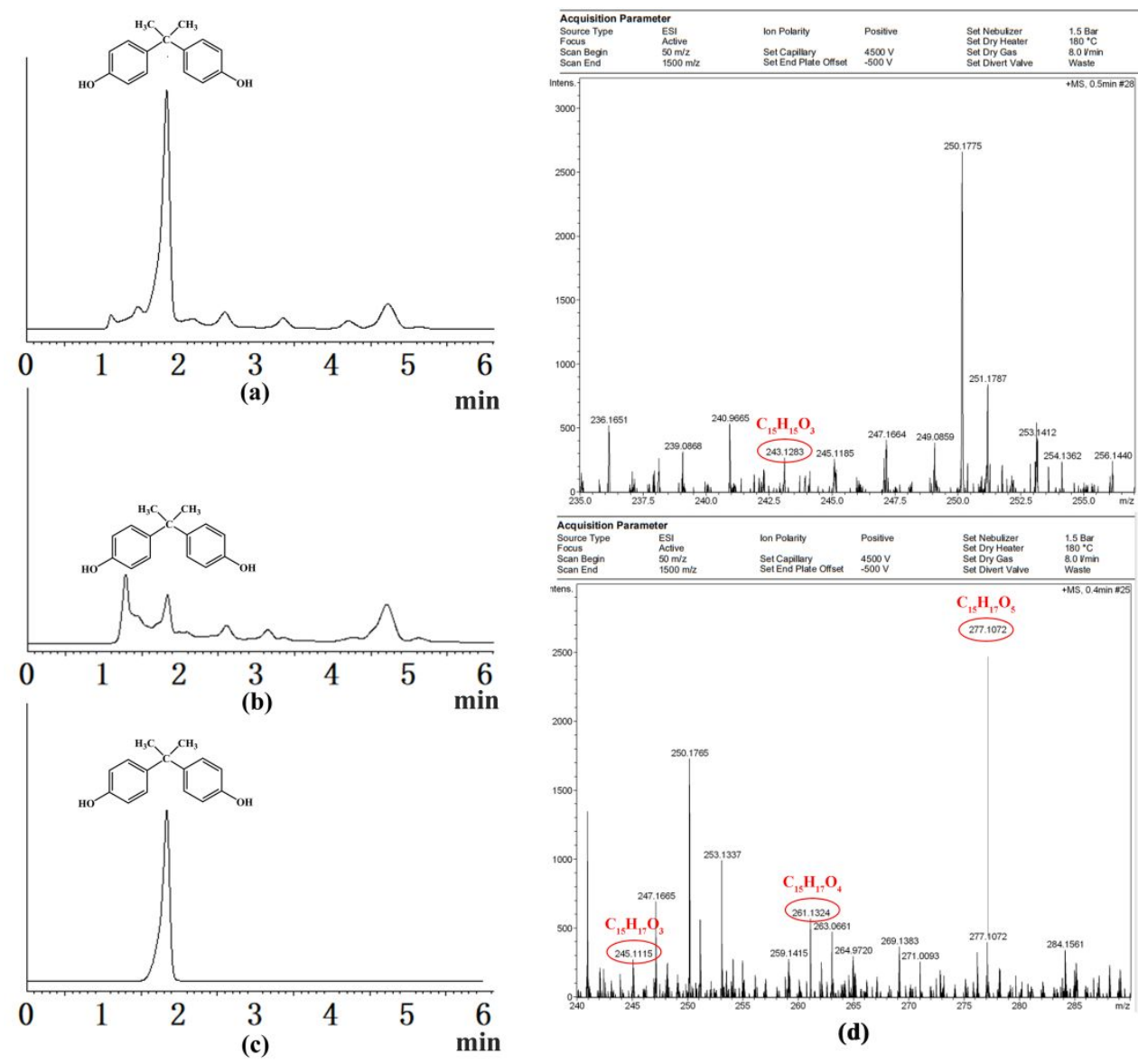

(d)
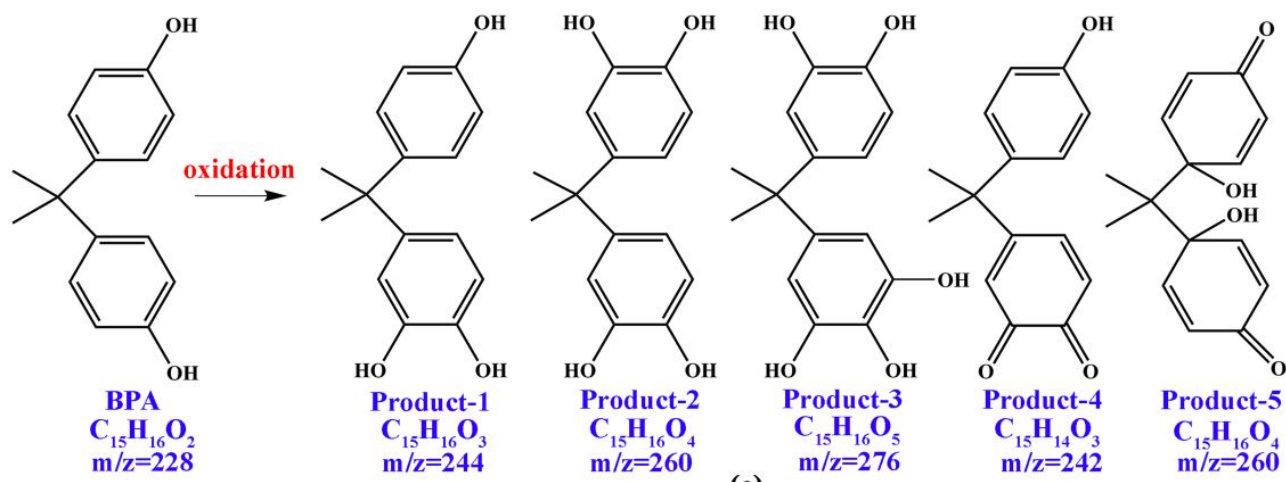

(e)

Figure S6. The high-performance liquid chromatography (HPLC) spectra of BPA oxidation products: (a) in the electrolyte, (b) on the electrode, and (c) BPA in mobile phase of $\mathrm{MeOH}$ and $\mathrm{H}_{2} \mathrm{O}(85: 15)$ (experimental conditions: [BPA] $=50 \mu \mathrm{M}$, electrolysis time 3h); ESI-MS spectra of the oxidation products of BPA (d). Combined with HPLC spectra (a-c) and ESI-MS spectra (d). The electro-catalytic oxidation of BPA on Zn-L-IPA@GP/GCE probably produced a mixture of multiple products. The products were deduced to be hydroxylated-BPA and quinones products, ${ }^{\mathrm{S} 11, \mathrm{~S} 12}$ as shown in the Figure S6(e). 

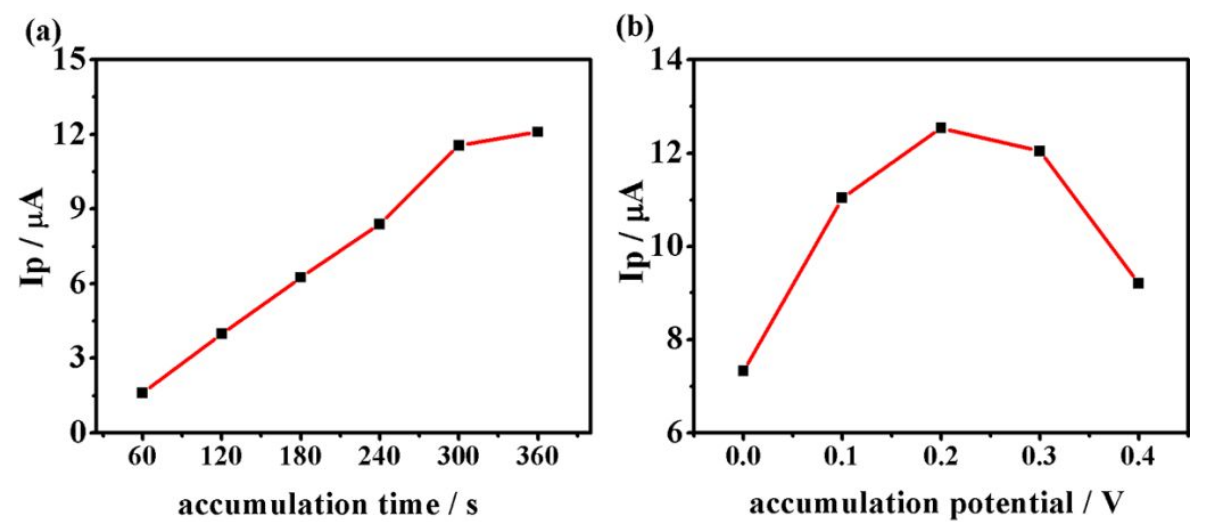

Figure S7. Influence of accumulation times (a) and accumulation potentials (b) on $I_{\mathrm{p}}$ of BPA.
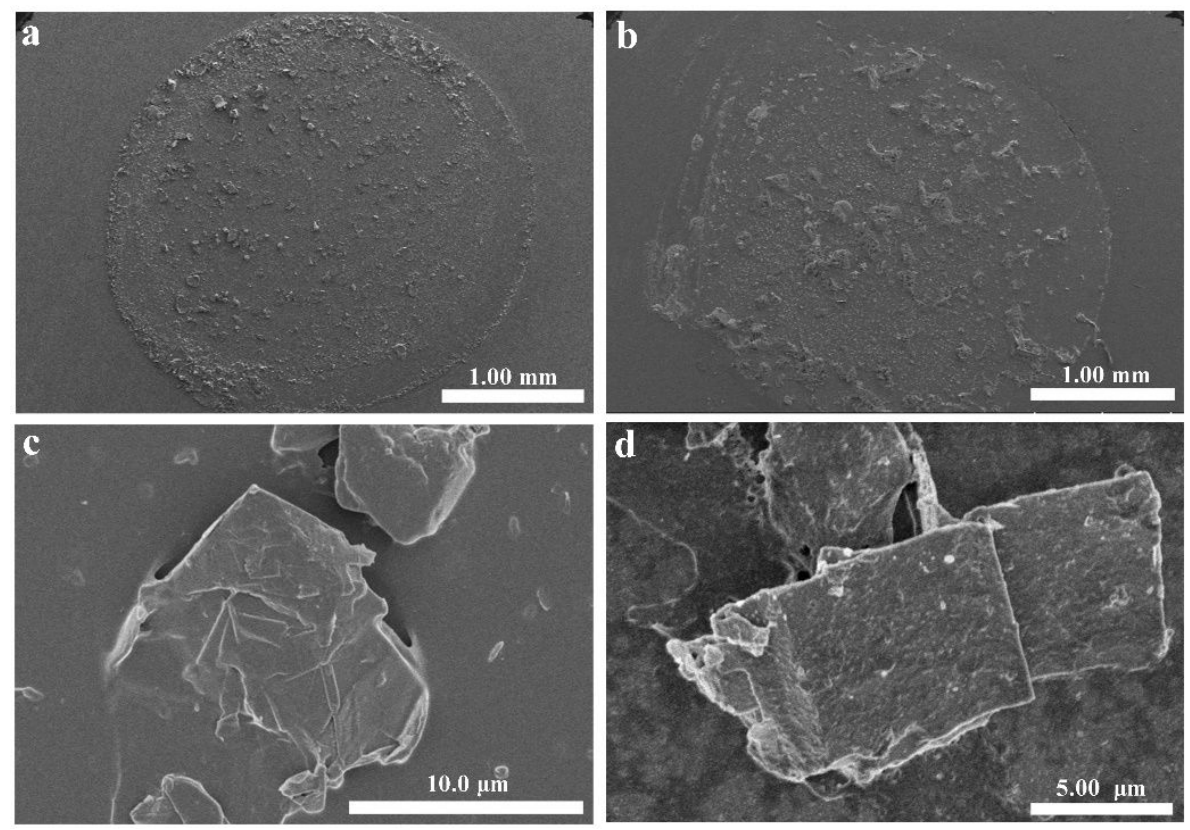

Figure S8. SEM images of Zn-L-IPA@GP/GCE surface before (a, c) and after (b, d) tested every day within a week. 


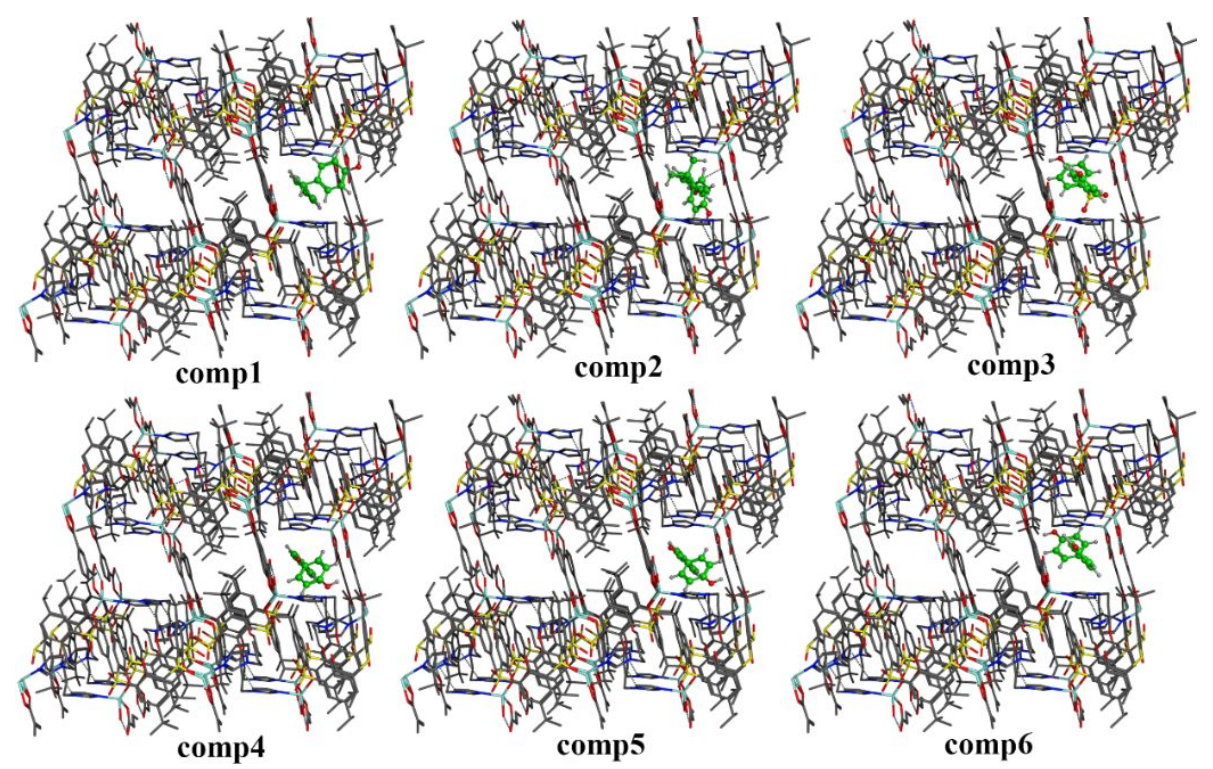

Figure S9. Host-guest complexes formed by the docking of comp1-comp6 with Zn-L-IPA. 
Table S1. Crystallographic Data for Zn-L-IPA.

\begin{tabular}{ll}
\hline Compound & $\mathrm{Zn}-\mathrm{L}-\mathrm{IPA}$ \\
\hline Formula & $\mathrm{Zn}_{2} \mathrm{C}_{68} \mathrm{H}_{96} \mathrm{~N}_{6} \mathrm{O}_{24} \mathrm{~S}_{4}$ \\
$M r$ & 1640.48 \\
Temperature $(\mathrm{K})$ & $173(2) \mathrm{K}$ \\
Crystal system & Triclinic \\
Space group & $P-1$ \\
$a(\AA)$ & $15.5034(15)$ \\
$b(\AA)$ & $15.6399(15)$ \\
$c(\AA)$ & $15.7105(16)$ \\
$\alpha\left(^{\circ}\right)$ & $89.983(6)$ \\
$\beta\left(^{\circ}\right)$ & $86.717(6)$ \\
$\left.\gamma^{\circ}\right)$ & $75.247(5)$ \\
$V\left(\AA^{3}\right)$ & $3677.3(6)$ \\
$Z$ & 2 \\
$D_{\text {calc }}\left(\mathrm{g} \cdot \mathrm{cm}{ }^{-3}\right)$ & 1.482 \\
$F(000)$ & 1724 \\
$R_{\text {int }}$ & 0.0613 \\
$\mathrm{GOF}$ on $F^{2}$ & 1.156 \\
${ }^{a} R_{1}$ & 0.0805 \\
${ }^{b} w R_{2}[\mathrm{I}>2 \sigma(\mathrm{I})]$ & 0.2421 \\
\hline${ }^{a} R_{1}=\sum|| F_{o}|-| F_{c}|| \sum\left|F_{o}\right|,{ }^{\circ} w R_{2}$ & $\left\{\sum \mathrm{w}\left[\left(F_{o}\right)^{2}-\left(F_{c}\right)^{2}\right]^{2} / \sum \mathrm{w}\left[\left(F_{o}\right)^{2}\right]^{2}\right\}^{1 / 2}$ \\
&
\end{tabular}


Table S2. Selected Bond Distances $(\AA)$ and Angles (deg) for Zn-L-IPA.

\begin{tabular}{llll}
\hline & \multicolumn{3}{l}{$\mathrm{Zn}-\mathrm{L}-\mathrm{IPA}$} \\
\hline $\mathrm{Zn}(1)-\mathrm{O}(14)$ & $1.877(3)$ & $\mathrm{Zn}(1)-\mathrm{O}(11)$ & $1.958(5)$ \\
$\mathrm{Zn}(1)-\mathrm{N}(2)^{\# 1}$ & $1.986(5)$ & $\mathrm{Zn}(1)-\mathrm{O}(1 \mathrm{~W})$ & $2.060(6)$ \\
$\mathrm{Zn}(2)-\mathrm{O}(1)$ & $1.871(4)$ & $\mathrm{Zn}(2)-\mathrm{N}(3)^{\# 2}$ & $1.945(5)$ \\
$\mathrm{Zn}(2)-\mathrm{O}(7)^{\# 3}$ & $1.967(4)$ & $\mathrm{Zn}(2)-\mathrm{O}(16)^{\# 3}$ & $2.332(6)$ \\
$\mathrm{Zn}(2)-\mathrm{O}(6)$ & $2.361(6)$ & $\mathrm{Zn}\left(2^{\prime}\right)-\mathrm{O}(16)^{\# 3}$ & $1.870(8)$ \\
$\mathrm{Zn}\left(2^{\prime}\right)-\mathrm{O}(1)$ & $1.812(6)$ & $\mathrm{Zn}\left(2^{\prime}\right)-\mathrm{O}(7)^{\# 3}$ & $2.354(9)$ \\
$\mathrm{Zn}\left(2^{\prime}\right)-\mathrm{N}(3)^{\# 2}$ & $2.109(8)$ & $\mathrm{O}(14)-\mathrm{Zn}(1)-\mathrm{O}(11)$ & $124.43(18)$ \\
$\mathrm{O}(14)-\mathrm{Zn}(1)-\mathrm{N}(2)^{\# 1}$ & $116.92(19)$ & $\mathrm{O}(11)-\mathrm{Zn}(1)-\mathrm{N}(2)^{\# 1}$ & $106.9(2)$ \\
$\mathrm{O}(14)-\mathrm{Zn}(1)-\mathrm{O}(1 \mathrm{~W})$ & $105.43(18)$ & $\mathrm{O}(11)-\mathrm{Zn}(1)-\mathrm{O}(1 \mathrm{~W})$ & $94.8(2)$ \\
$\mathrm{N}(2)^{\# 1}-\mathrm{Zn}(1)-\mathrm{O}(1 \mathrm{~W})$ & $103.9(2)$ & $\mathrm{O}(1)-\mathrm{Zn}(2)-\mathrm{N}(3)^{\# 2}$ & $126.1(2)$ \\
$\mathrm{O}(1)-\mathrm{Zn}(2)-\mathrm{O}(7)^{\# 3}$ & $122.47(18)$ & $\mathrm{N}(3)^{\# 2}-\mathrm{Zn}(2)-\mathrm{O}(7)^{\# 3}$ & $111.2(2)$ \\
$\mathrm{O}(1)-\mathrm{Zn}(2)-\mathrm{O}(16)^{\# 3}$ & $106.5(2)$ & $\mathrm{N}(3)^{\# 2}-\mathrm{Zn}(2)-\mathrm{O}(16)^{\# 3}$ & $95.7(2)$ \\
$\mathrm{O}(7)^{\# 3}-\mathrm{Zn}(2)-\mathrm{O}(16)^{\# 3}$ & $60.0(2)$ & $\mathrm{O}(1)-\mathrm{Zn}(2)-\mathrm{O}(6)$ & $85.03(17)$ \\
$\mathrm{N}(3)^{\# 2}-\mathrm{Zn}(2)-\mathrm{O}(6)$ & $89.3(2)$ & $\mathrm{O}(7)^{\# 3}-\mathrm{Zn}(2)-\mathrm{O}(6)$ & $100.9(2)$ \\
$\mathrm{O}(16)^{\# 3}-\mathrm{Zn}(2)-\mathrm{O}(6)$ & $160.8(2)$ & $\mathrm{O}(1)-\mathrm{Zn}\left(2^{\prime}\right)-\mathrm{O}(16)^{\# 3}$ & $133.1(3)$ \\
$\mathrm{O}(1)-\mathrm{Zn}\left(2^{\prime}\right)-\mathrm{N}(3)^{\# 2}$ & $120.2(4)$ & $\mathrm{O}(16)^{\# 3}-\mathrm{Zn}\left(2^{\prime}\right)-\mathrm{N}(3)^{\# 2}$ & $106.0(3)$ \\
$\mathrm{O}(1)-\mathrm{Zn}\left(2^{\prime}\right)-\mathrm{O}(7)^{\# 3}$ & $107.0(4)$ & $\mathrm{O}(16)^{\# 3}-\mathrm{Zn}\left(2^{\prime}\right)-\mathrm{O}(7)^{\# 3}$ & $60.6(3)$ \\
$\mathrm{N}(3)^{\# 2}-\mathrm{Zn}\left(2^{\prime}\right)-\mathrm{O}(7)^{\# 3}$ & $92.5(4)$ & & \\
\hline $\mathrm{Sym}$ & & & \\
\hline
\end{tabular}

Symmetry transformations used to generate equivalent atoms: ${ }^{\# 1} 1-\mathrm{x}, 1-\mathrm{y}, 1-\mathrm{z}$; ${ }^{\# 2} 1-\mathrm{x}$, 2-y, -z; ${ }^{\# 3}-\mathrm{x}, 2-\mathrm{y}, 1-\mathrm{z}$.

Table S3. Hydrogen Bond Distances $(\AA)$ and Angles (deg) for Zn-L-IPA.

\begin{tabular}{lllll}
\hline $\mathrm{D}-\mathrm{H} \cdots \mathrm{A}$ & $\mathrm{d}(\mathrm{D}-\mathrm{H})$ & $\mathrm{d}(\mathrm{H} \cdots \mathrm{A})$ & $\mathrm{d}(\mathrm{D} \cdots \mathrm{A})$ & $<(\mathrm{DHA})$ \\
\hline $\mathrm{C} 44-\mathrm{H} 44 \cdots \mathrm{O}^{\# 1}$ & 0.95 & 2.57 & $3.332(8)$ & 137.3 \\
$\mathrm{C} 46-\mathrm{H} 46 \cdots \mathrm{O}^{\# 2}$ & 0.95 & 2.49 & $3.014(9)$ & 114.9 \\
$\mathrm{C} 41-\mathrm{H} 41 \cdots \mathrm{O}^{\# 4}$ & 0.99 & 2.77 & 3.217 & 107.98 \\
\hline
\end{tabular}

Symmetry code for Zn-L-IPA: ${ }^{\# 1} 1-x, 1-y, 1-z ;{ }^{\# 2} 1-x, 2-y,-z ;{ }^{\# 4} 1-x, 1-y,-z$. 
Table S4. The linear regression equations of BPA and BPF on Zn-L-IPA@GP/GCE.

\begin{tabular}{cllc}
\hline Bisphenols & $\begin{array}{c}\text { Linear range } \\
(\mu \mathrm{M})\end{array}$ & Linear regression equation & $\mathrm{R}^{2}$ \\
\hline BPA & $0.01-0.5$ & $I_{\mathrm{pa}}(\mu \mathrm{A})=0.272 \mathrm{C}(\mu \mathrm{M})+0.052$ & 0.992 \\
BPA & $0.5-8.5$ & $I_{\mathrm{pa}}(\mu \mathrm{A})=0.019 \mathrm{C}(\mu \mathrm{M})+0.177$ & 0.998 \\
BPF & $0.005-0.75$ & $I_{\mathrm{pa}}(\mu \mathrm{A})=0.484 \mathrm{C}(\mu \mathrm{M})+0.038$ & 0.994 \\
BPF & $0.75-10.5$ & $I_{\mathrm{pa}}(\mu \mathrm{A})=0.016 \mathrm{C}(\mu \mathrm{M})+0.380$ & 0.992 \\
\hline
\end{tabular}


Table S5. Analytical performances of reported electrochemical sensors for BPA and BPF.

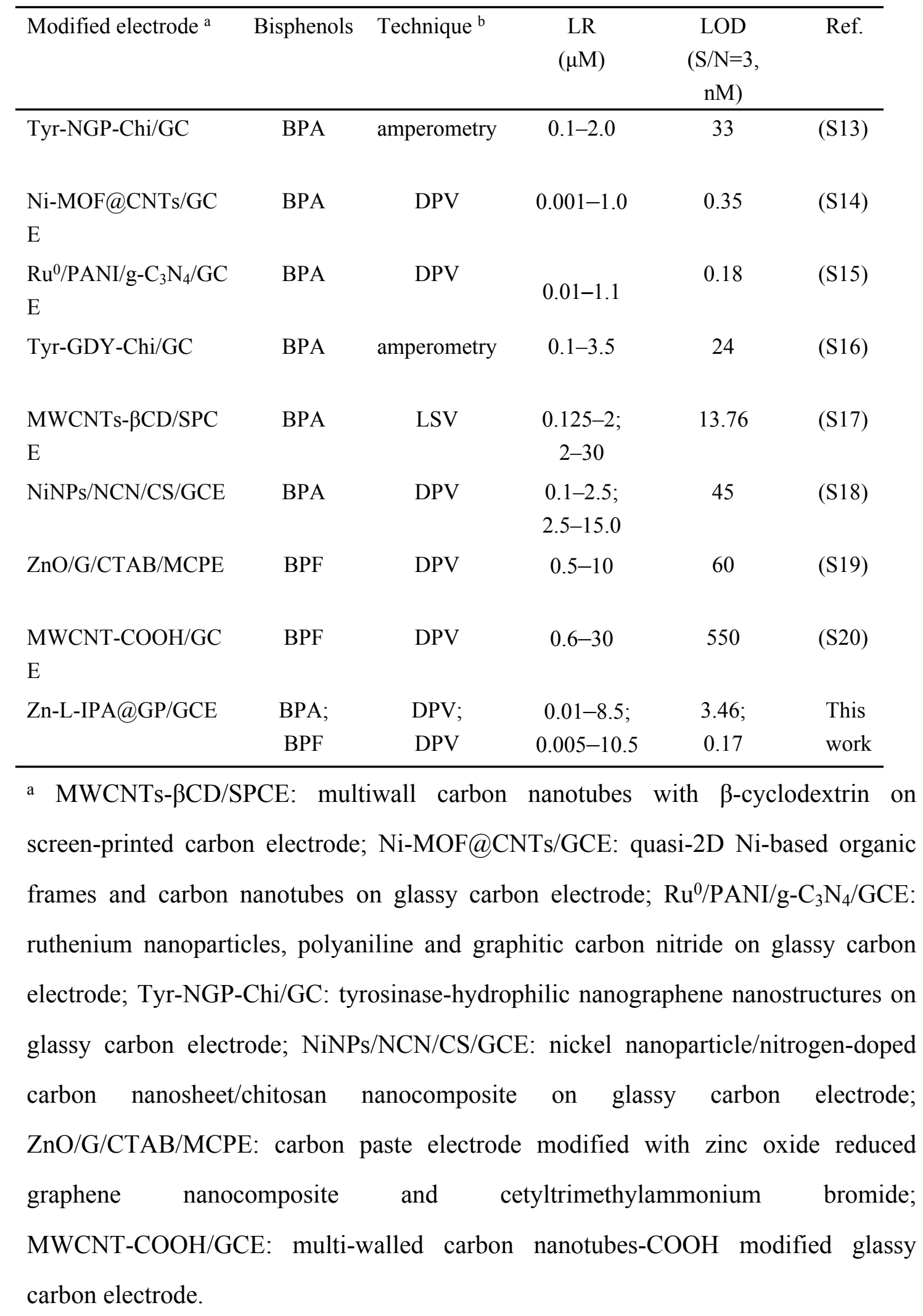


b DPV: differential pulse voltammetry; LSV: linear sweep voltammetry.

Table S6. Assessment of BPA and BPF recovery efficiencies on

Zn-L-IPA@GP/GCE in various real samples $(\mathrm{n}=3)$.

\begin{tabular}{lcccccccc}
\hline $\begin{array}{l}\text { Water } \\
\text { samples }\end{array}$ & BPA & \multicolumn{7}{c}{ BPF } \\
\cline { 2 - 9 } & $\begin{array}{c}\text { Add } \\
(\mu \mathrm{M})\end{array}$ & $\begin{array}{c}\text { Found } \\
(\mu \mathrm{M})\end{array}$ & $\begin{array}{c}\text { Recovery } \\
(\%)\end{array}$ & $\begin{array}{c}\text { R.S.D } \\
(\%)\end{array}$ & $\begin{array}{c}\text { Add } \\
(\mu \mathrm{M})\end{array}$ & $\begin{array}{c}\text { Found } \\
(\mu \mathrm{M})\end{array}$ & $\begin{array}{c}\text { Recovery } \\
(\%)\end{array}$ & $\begin{array}{c}\text { R.S.D } \\
(\%)\end{array}$ \\
\hline \multirow{2}{*}{ Tap } & 0.5 & $0.48 \pm 0.01$ & $96.0 \pm 2.0$ & 2.12 & 0.5 & $0.49 \pm 0.01$ & $98.0 \pm 2.0$ & 1.99 \\
& 5.0 & $4.94 \pm 0.13$ & $98.8 \pm 2.6$ & 2.28 & 5.0 & $4.98 \pm 0.19$ & $99.6 \pm 3.8$ & 2.37 \\
\multirow{2}{*}{ Mineral } & 0.5 & $0.49 \pm 0.01$ & $98.0 \pm 2.0$ & 1.89 & 0.5 & $0.49 \pm 0.01$ & $98.0 \pm 2.0$ & 0.99 \\
& 5.0 & $4.97 \pm 0.19$ & $99.4 \pm 3.8$ & 1.85 & 5.0 & $4.88 \pm 0.15$ & $97.6 \pm 3.0$ & 2.38 \\
\cline { 1 - 6 } Lake & 0.5 & $0.49 \pm 0.01$ & $98.0 \pm 2.0$ & 2.56 & 0.5 & $0.50 \pm 0.01$ & $100.0 \pm 2.0$ & 3.04 \\
& 5.0 & $4.89 \pm 0.08$ & $97.8 \pm 1.6$ & 1.77 & 5.0 & $5.02 \pm 0.12$ & $100.4 \pm 2.4$ & 2.75 \\
\hline
\end{tabular}

\section{REFERENCES}

(S1) Xu, L.; Yang, J.; Liu, Y.-Y.; Xu, G.-H.; Ma, J.-F. Structures and luminescent properties of five $\mathrm{Cd}(\mathrm{II})$ metal-organic frameworks assembled by imidazole-based thiacalix[4]arene ligand and ancillary dicarboxylic acids. J. Lumin. 2019, 209, 398-403.

(S2) Iki, N.; Kumagai, H.; Morohashi, N.; Ejima, K.; Hasegawa, M.; Miyanari, S. Miyano, S. Selective oxidation of thiacalix[4]arenes to the sulfinyl- and sulfonylcalix[4]arenes and their coordination ability to metal ions. Tetrahedron Lett. 1998, 39,7559-7562.

(S3) Sheldrick, G. M. SHELXTL-2018/3, Program for the Crystal Structure Solution; University of Göttingen: Göttingen, Germany, 2018.

(S4) Sheldrick, G. M. SHELXTL-2018/3, Program for the Crystal Structure Refinement; University of Göttingen: Göttingen, Germany, 2018.

(S5) Zhang, Y.; Sanner, M. F. Docking Flexible Cyclic Peptides with AutoDock CrankPep. J. Chem. Theory Comput. 2019, 15, 5161-5168.

(S6) Morris, G. M.; Huey, R.; Lindstrom, W.; Sanner, M. F.; Belew, R. K.; Goodsell, D. S.; Olson, A. J. AutoDock4 and AutoDockTools4: Automated Docking with Selective Receptor Flexibility. J. Comput. Chem. 2009, 30, 2785-2791. 
(S7) Bramucci, E.; Paiardini, A.; Bossa, F.; Pascarella, S. PyMod: sequence similarity searches, multiple sequence-structure alignments, and homology modeling within PyMOL. BMC Bioinformatics 2012, 13, Suppl 4.

(S8) Yang, L.; Li, D.; Guo, B.; Wei, D. Theoretical study on the inclusion interaction of $\beta$-cyclodextrin with gabapentin and its stability. J. Struct. Chem. 2019, 60, 564574.

(S9) Tirado-Rives J.; Jorgensen, W. L. Performance of B3LYP Density Functional Methods for a Large Set of Organic Molecules. J. Chem. Theory Comput. 2008, 4, 297-306.

(S10) Frisch, M. J.; Trucks, G. W.; Schlegel, H. B.; Scuseria, G. E.; Robb, M. A.; Cheeseman, J. R.; Scalmani, G.; Barone, V.; Mennucci, B.; Petersson, G. A.; Nakatsuji, H.; Caricato, M.; Li, X.; Hratchian, H. P.; Izmaylov, A. F.; Bloino, J.; Zheng, G.; Sonnenberg, J. L.; Hada, M.; Ehara, M.; Toyota, K.; Fukuda, R.; Hasegawa, J.; Ishida, M.; Nakajima, T.; Honda, Y.; Kitao, O.; Nakai, H.; Vreven, T.; Montgomery, J. A.,Jr.; Peralta, J. E.; Ogliaro, F.; Bearpark, M.; Heyd, J. J.; Brothers, E.; Kudin, K. N.; Staroverov, V. N.; Kobayashi, R.; Normand, J.; Raghavachari, K.; Rendell, A.; Burant, J. C.; Iyengar, S. S.; Tomasi, J.; Cossi, M.; Rega, N.; Millam, J. M.; Klene, M.; Knox, J. E.; Cross, J. B.; Bakken, V.; Adamo, C.; Jaramillo, J.; Gomperts, R.; Stratmann, R. E.; Yazyev, O.; Austin, A. J.; Cammi, R.; Pomelli, C.; Ochterski, J. W.; Martin, R. L.; Morokuma, K.; Zakrzewski, V. G.; Voth, G. A.; Salvador, P.; Dannenberg, J. J.; Dapprich, S.; Daniels, A. D.; Farkas, Ö.; Foresman, J. B.; Ortiz, J. V.; Cioslowski, J.; Fox, D. J. Gaussian, Inc., Wallingford CT 2009.

(S11) You, J.; Zhang, C.; Wu, Z.; Ao, Z.; Sun, W.; Xiong, Z.; Su, S.; Yao, G.; Lai, B. N-doped graphite encapsulated metal nanoparticles catalyst for removal of Bisphenol A via activation of peroxymonosulfate: A singlet oxygen-dominated oxidation process. Chem. Eng. J. 2021, 415, 128890.

(S12) Wu, W.; Huang, Z.-H.; Lim, T.-T. A comparative study on electrochemical oxidation of bisphenol A by boron-doped diamond anode and modified $\mathrm{SnO}_{2}-\mathrm{Sb}$ anodes: Influencing parameters and reaction pathways. J. Environ. Chem. Eng. 2016, 
4, 2807-2815.

(S13) Wu, L.; Deng, D.; Jin, J.; Lu, X.; Chen, J. Nanographene-based tyrosinase biosensor for rapid detection of bisphenol A. Biosens. Bioelectron. 2012, $35,193-199$.

(S14) Xu, C.; Liu, L.; Wu, C.; Wu, K. Unique 3D heterostructures assembled by quasi-2D Ni-MOF and CNTs for ultrasensitive electrochemical sensing of bisphenol A. Sens. Actuators B: Chem. 2020, 310, 127885.

(S15) Ponnaiah, S. K.; Prakash, P.; Muthupandian, S. Ultrasonic energy-assisted in-situ synthesis of $\mathrm{Ru}^{0} / \mathrm{PANI} / \mathrm{g}-\mathrm{C}_{3} \mathrm{~N}_{4}$ nanocomposite: Application for picomolar-level electrochemical detection of endocrine disruptor (Bisphenol-A) in humans and animals. Ultrason. Sonochem. 2019, 58, 104629.

(S16) Wu, L.; Gao, J.; Lu, X.; Huang, C.; Dhanjai; Chen, J. Graphdiyne: A new promising member of $2 \mathrm{D}$ all-carbon nanomaterial as robust electrochemical enzyme biosensor platform. Carbon 2020, 156, 568-575.

(S17) Ali, M. Y.; Alam, A. U.; Howlader, M. M. R. Fabrication of highly sensitive Bisphenol A electrochemical sensor amplified with chemically modified multiwall carbon nanotubes and $\beta$-cyclodextrin. Sens. Actuators B: Chem. 2020, 320, 128319.

(S18) Wang, Y.; Yin, C.; Zhuang, Q. An electrochemical sensor modified with nickel nanoparticle/nitrogen-doped carbon nanosheet nanocomposite for bisphenol A detection. J. Alloys Compd. 2020, 827,154335.

(S19) Manasa, G.; Mascarenhas, R. J.; Satpati, A. K.; Basavaraja, B. M.; Kumar, S. An electrochemical Bisphenol F sensor based on $\mathrm{ZnO} / \mathrm{G}$ nano composite and CTAB surface modified carbon paste electrode architecture. Colloids Surf. B Biointerfaces 2018, 170, 144-151.

(S20) Wang, X.; Yang, L.; Jin, X.; Zhang, L. Electrochemical determination of estrogenic compound bisphenol $\mathrm{F}$ in food packaging using carboxyl functionalized multi-walled carbon nanotubes modified glassy carbon electrode. Food Chem. 2014, $157,464-469$. 